\title{
Midfrontal theta tracks action monitoring over multiple interactive time scales
}

\author{
Michael X Cohen \\ Radboud University and Radboud University Medical Center, The Netherlands
}

\section{A R T I C L E I N F O}

Article history:

Received 25 April 2016

Accepted 26 July 2016

Available online 27 July 2016

Keywords:

Cognitive control

Oscillations

Theta

Scale-free

Criticality

Action monitoring

Long-range temporal autocorrelations

\begin{abstract}
A B S T R A C T
Quickly detecting and correcting mistakes is a crucial brain function. EEG studies have identified an idiosyncratic electrophysiological signature of online error correction, termed midfrontal theta. Midfrontal theta has so far been investigated over the fast time-scale of a few hundred milliseconds. But several aspects of behavior and brain activity unfold over multiple time scales, displaying "scale-free" dynamics that have been linked to criticality and optimal flexibility when responding to changing environmental demands. Here we used a novel line-tracking task to demonstrate that midfrontal theta is a transient yet non-phase-locked response that is modulated by task performance over at least three time scales: a few hundred milliseconds at the onset of a mistake, task performance over a fixed window of the previous $5 \mathrm{~s}$, and scale-free-like fluctuations over many tens of seconds. These findings provide novel evidence for a role of midfrontal theta in online behavioral adaptation, and suggest new approaches for linking EEG signatures of human executive functioning to its neurobiological underpinnings.
\end{abstract}

(C) 2016 Published by Elsevier Inc.

\section{Introduction}

Biological and social success in a complex and rapidly changing world requires the ability to monitor one's actions and engage fast but effective adjustments to avoid or correct mistakes. The neural computations underlying action monitoring and how those computations are implemented by neural circuits remain under-explored and largely speculative (Cohen, 2014a). One promising finding is theta-band oscillations $(\sim 6 \mathrm{~Hz})$ in the medial prefrontal cortex observed during action monitoring tasks, in particular, tasks that elicit conflict between multiple competing responses (Cohen and Donner, 2013; Huster et al., 2013; Nigbur et al., 2012; Oehrn et al., 2014). This action monitoringrelated increase in midfrontal theta is seen using a variety of experimental tasks (e.g., Stroop, flankers, Simon), data analysis methods, and spatial and temporal filters. It does not reflect band-pass filtering an ERP or other non-oscillatory transient (Cohen and Donner, 2013). It has high statistical power ( $>0.9$ with 18 subjects) and correlates both with single-trial performance (as measured through reaction times) and with individual differences in the ability to adapt behavior after errors or learning from negative feedback (Cohen, 2011; Nakao et al., 2012). Some of these features have also been observed in non-human animals (Narayanan et al., 2013; Womelsdorf et al., 2010). In order words, midfrontal theta is a robust and sensitive marker for the recruitment of the brain's action monitoring system.

Action monitoring-related midfrontal theta may provide a link between cognitive psychological studies of human decision-making and

E-mail address: mikexcohen@gmail.com. in-vivo and in-vitro neurophysiology studies of oscillations in lower animals and in computational models. Indeed, neural oscillations are evident across many spatial and temporal scales of the brain (Donner and Siegel, 2011; Le Van Quyen et al., 2013; Varela et al., 2001), and the underlying biophysical mechanisms of oscillations are increasingly well-understood (Wang, 2010). Among other functions, oscillations are thought to provide a temporal structure for local and long-range neural communication in the brain (Akam and Kullmann, 2012; Buzsáki and Moser, 2013; Fries, 2005).

Research on midfrontal theta has been performed exclusively using discrete trial-based tasks that elicit midfrontal theta over the timescale of a few hundred milliseconds. It is unknown whether the phasic nature of midfrontal theta is related to the phasic trials in computerbased tasks, or whether this reflects the phasic nature of the neural computations underlying action monitoring.

Furthermore, many aspects of behavior and brain activity occur over multiple time-scales in a manner consistent with scale-free or fractal dynamics (Kello et al., 2010). "Scale-free behavior" refers to time series that lack a characteristic time-scale. Such behavior was initially observed in physical systems like sand piles and Earthquake magnitudes. The discovery that similar scaling laws are present in biological systems including the human brain has led to ideas about shared fundamental principles that may govern both physical and biological phenomena, in particular as it relates to complexity and system stability. Debate remains in the literature as to how precisely to quantify evidence for scale-free organization, and further debate concerns whether a scalefree organization has any functional significance (Beggs and Timme, 2012; Farrell et al., 2006). Indeed, even if the brain exhibits 
characteristics consistent with a scale-free organization, this organization is merely a curious epiphenomenon if it does not constrain or relate to cognition or neural computations. However, increasing evidence suggests that scaling properties of the brain are in fact relevant for brain function: Scaling exponents are correlated, across subjects, with clinical symptoms such as Alzheimer's disease severity (Montez et al., 2009), and with performance in peri-threshold sensory detection tasks (Palva et al., 2013) and memory tasks (Stokić et al., 2015).

A complex system (e.g., the brain) operating at or near a critical point is maximally flexible and able to adjust its responses to various external demands (Chialvo, 2010; Linkenkaer-Hansen et al., 2001). Given that midfrontal theta is consistently linked to rapid and flexible adjustments of behavior, it seems natural to wonder whether midfrontal theta exhibits properties consistent with a scale-free system, or whether midfrontal theta is modulated by scale-free properties in behavior.

The purpose of this study was to examine whether error monitoring and online action adjustments operate over a range of time scales consistent with a scale-free-like organization; and if so, how such behavior might be related to midfrontal theta. In particular, the main analyses were focused on whether the phasic midfrontal theta activation to response errors and their correction is related to temporal scaling properties of behavior. Exploratory analyses were also conducted to examine how scaling exponents of neural oscillations were related to scaling exponents of behavior over a wider range of frequencies and topographical regions.

To study scale-free-like dynamics in behavior, the discrete trial approach that dominates empirical research in cognitive neuroscience may be suboptimal. In part this is because neurocognitive processes might be "reset" by the staccato nature of discrete-trial task designs, and in part because neural processes may become entrained to the task structure (typically around 0.5 to $1 \mathrm{~Hz}$ ). These features might not impede investigations into scale-free dynamics over long time-scales (minutes to hours), but they impede understanding faster time-scale dynamics, for example in the range of seconds to tens of seconds. More relevant for studying cognitive control, action monitoring tasks typically involve different trial types (e.g., incongruent vs. congruent trials) that are pseudo-randomly intermixed, and this mixing may "reset" or otherwise interfere with dynamics at time scales of seconds to tens of seconds. Indeed, it is well-known that response conflicts and errors in cognitive control tasks induce trial-sequence effects that affect behavior for many seconds (Egner et al., 2010; Egner, 2007). Therefore, this research involved developing a new experimental paradigm that might be better suited for examining long-range temporal correlations in behavior and linking them to brain activity over multiple time scales.

\section{Materials and methods}

\section{Human participants}

Twenty-one right-handed healthy adults ( 12 females) participated in this experiment, all with normal or corrected-to-normal vision. Age ranged from 20 to 33 years $(M=24.7 ; S D=3.3$ ). Subjects were current or former students from the University of Amsterdam. They gave informed consent according to an ethics protocol approved by the ethics committee at the department of psychology. Subjects were compensated with course credits or a monetary reward of 10.50 Euros. Data from two male participants were excluded from the analysis due to excessive EEG artifacts.

\section{Experiment and behavioral data}

In the continuous action adjustment task (programmed using Presentation software; neurobs.com), subjects were instructed to move a disk on the computer screen in order to keep the disk on top of a moving line (Fig. 1A). The disk was centered on the screen and its y-axis position was controlled using a trackpad. Whenever the disk did not touch the line, its color would turn from green to red. The diameter of the disk adjusted to the level of performance, such that relatively poor performance increased the disk diameter-thus making the tracking easier-and vice-versa for better performance. An absolute minimum radius of 6 pixels, and an absolute maximum radius of 40 pixels, was imposed. The diameter of the disk reflected the subject's performance over a fixed sliding window of $5 \mathrm{~s}$; the more time the disk spent on/off the line, the smaller/bigger the disk became. The purposes of this feature were (1) to provide subjects with continuous visual feedback about their performance over the past $5 \mathrm{~s}$, and (2) to titrate task difficulty to make sure that subjects made sufficient errors during the task. Subjects were informed about the relationship between disk diameter and task performance, and were thus able to use this as an indicator of their short-term accuracy.

The task included two conditions, which were designed to facilitate a proactive strategy (subjects could visually predict where the line would go) or a reactive strategy (subjects could not predict the line's impending movements). These conditions are termed, respectively, "wavey" and "flat." In the wavey condition, the line moved in a smooth fashion defined by the sum of several sine waves that varied randomly but continuously over time. The effect of this manipulation was that it was possible to predict when the center point (where the disk was positioned) would move up vs. down. In the flat condition, the line was straight horizontal and moved up and down smoothly but with randomly varying speed and unpredictable direction-reversals.

Subjects sat on a comfortable office chair, with their eyes approximately $90 \mathrm{~cm}$ from the $1024 \times 76811^{\prime \prime}$ computer monitor (subjects were instructed to remain still but were not physically restrained). The track-pad was attached to the right armrest of the chair, via a velcro strap that was adjusted for each subject's comfort. After receiving instructions, they practiced the task for about 15 min while the EEG cap and electrodes were placed. The recording was conducted in a quiet, dimly lit setting, and lasted about $48 \mathrm{~min}$ (12 blocks of 4 min each, plus short self-paced breaks between blocks). Block condition alternated, with the condition of the first block being chosen randomly.

The primary behavioral dependent measure was accuracy, defined as whether the line was touching or not touching the disk. This accuracy was coded as +1 (on the line) or -1 (off the line) at each monitor refresh $(60 \mathrm{~Hz})$. Offline, these and other behavioral data were aligned with the EEG data markers and up-sampled to $512 \mathrm{~Hz}$ to facilitate direct comparison with the EEG data.

\section{EEG acquisition and preprocessing}

EEG data were recorded from 64 sites according to the international 10-20 system, using $\mathrm{Ag} / \mathrm{AgCl}$ surface electrodes of the Active-Two BioSemi system (BioSemi, Amsterdam, The Netherlands) (see www. biosemi.com for hardware details). Two additional electrodes were placed on the earlobes, and four periocular electrodes were used for the vertical and horizontal electro-oculogram (EOG). Electrode offsets were kept below $50 \mathrm{~K} \Omega$ and the signal was sampled at $512 \mathrm{~Hz}$.

All processing and analysis steps were conducted in Matlab R2015a using custom scripts and supplemented with tools from the eeglab toolbox (Delorme and Makeig, 2004). Continuous data were high-pass filtered at $0.1 \mathrm{~Hz}$ and re-referenced to the average of the 64 scalp electrodes. Manual rejection was used to identify and remove periods of large EEG artifacts. Task events used for time-locking the epoched data (transitions between correct and error) from two seconds before artifacts until two seconds after artifacts were removed; this ensured that peri-artifact EEG data were not used in the analyses. Independent components analysis was computed, and components clearly identifiable as capturing oculomotor, heart-beat, electromyogram, or other non-brain artifacts were removed; on average, 2.8 components were removed per subject. EEG and behavioral data can be downloaded from mikexcohen.com/data. 

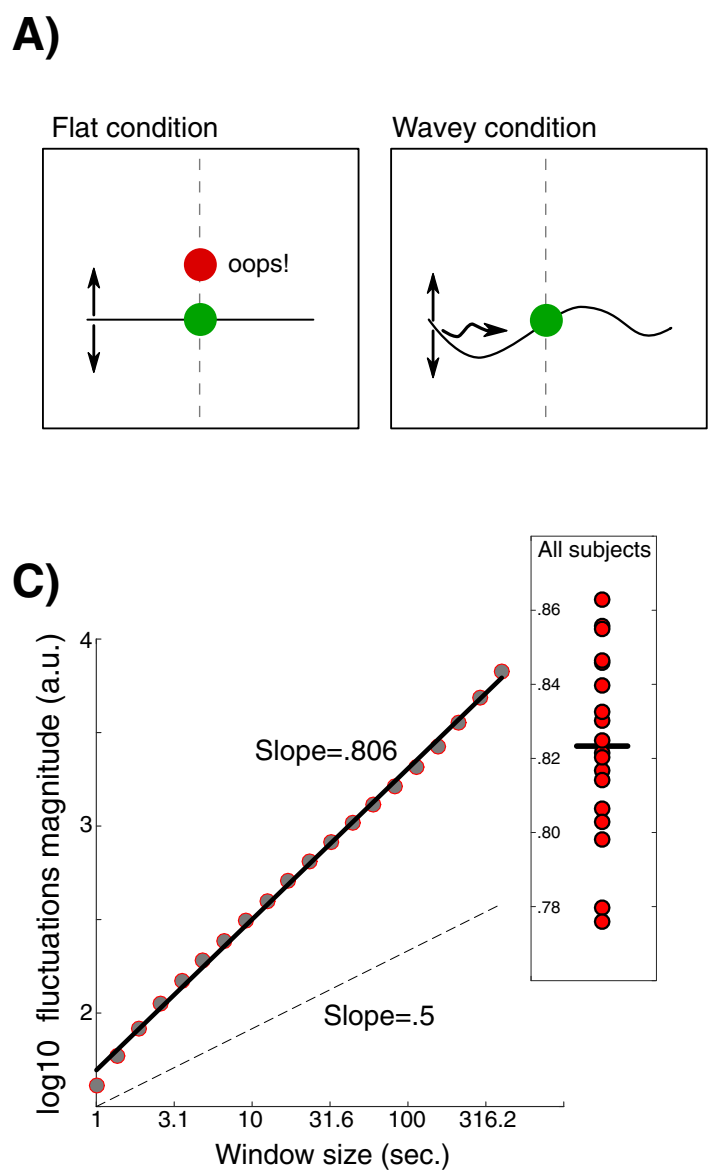

B)

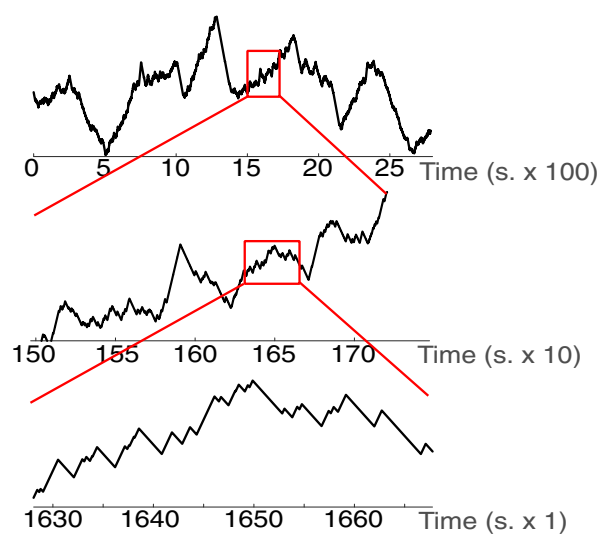

D)

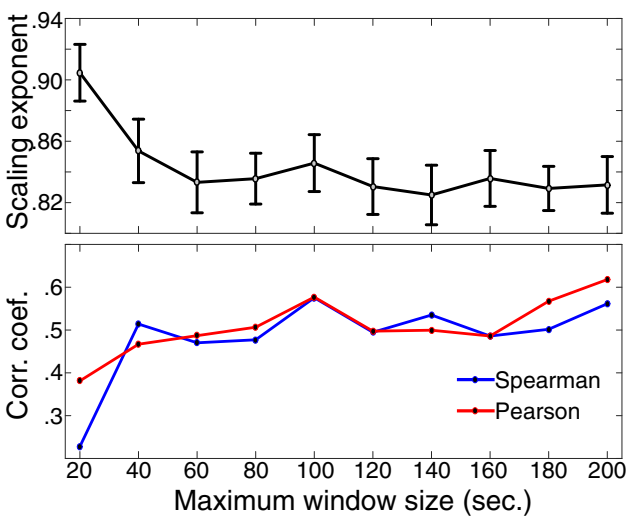

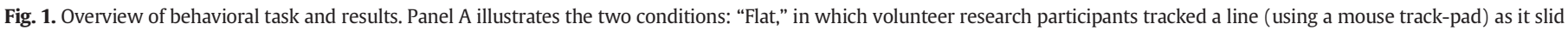

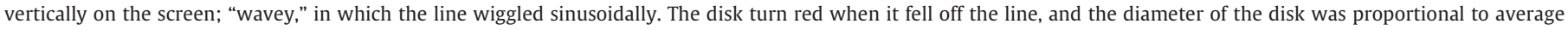

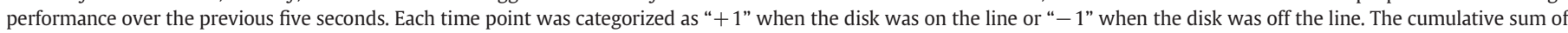

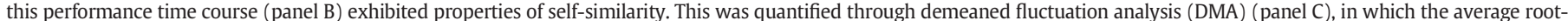

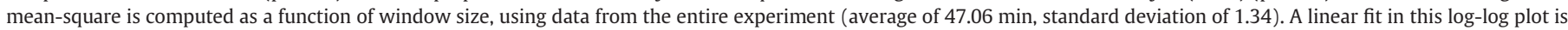

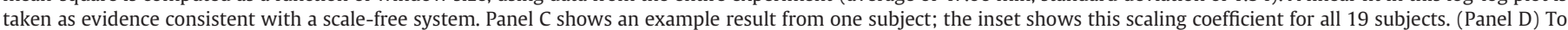

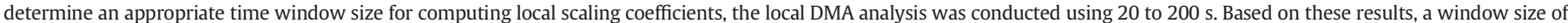
$60 \mathrm{~s}$ was used for subsequent analyses.

\section{EEG analyses}

Time-frequency decomposition was performed via complex Morlet wavelet convolution, which is detailed elsewhere (Cohen, 2014b). Forty frequencies between 1 and $80 \mathrm{~Hz}$ were extracted from the continuous data, using a logarithmically spaced number of cycles between 4 (at $1 \mathrm{~Hz}$ ) and 12 (at $80 \mathrm{~Hz}$ ). Continuous time series were epoched surrounding each behavioral transition (correct to error, and error to correct). The average number of epochs per subject per condition was 1283.00, 1283.47, 750.16, 749.16 (standard deviation: 300.06, 300.03, $115.44,116.88$ ), corresponding to conditions wavey error, wavey correct, flat error, flat correct (see also Fig. 2). The number of error and correct epochs are necessarily nearly perfectly matched because each correct epoch is the end of an error epoch, and vice-versa; slight differences resulted from epoch removal during data cleaning. Timefrequency power was taken as the squared magnitude of the result of complex convolution, and instantaneous phase angle was taken as the argument of the complex result. Inter-trial phase clustering is computed as the length of the average of the vectors at each time-frequency point over trials.

\section{Estimating global and local scaling exponents}

The scaling exponent was computed using the demeaned fluctuation analysis (DMA), which is comparable and, in some situations, preferable to, detrended fluctuation analysis (DFA) (Jiang and Zhou, 2011; Shao et al., 2012). Nonetheless, the two methods provide very similar results. The overall goal of DMA is to determine how the magnitude of time series fluctuations is related to the size of the time window in which those fluctuations are measured. The primary dependent measure of DMA and DFA is the scaling exponent, which measures the linear fit between the logarithm of the fluctuations and the logarithm of the time windows used to measure those fluctuations. Uncorrelated random noise with no long-range "memory" will have a scaling exponent of 0.5 , while processes with long-range positive temporal autocorrelations will have scaling exponents between 0.5 and 1 . Procedures for DMA and DFA are detailed in many other publications (e.g., Hardstone et al., 2012; Ihlen, 2012) and briefly described here. First, the data were de-meaned and integrated over time (as described below, the raw EEG signal was not integrated; the power time series extracted from wavelet convolution was integrated). Next, for each of 20 logarithmically spaced scales between one and $400 \mathrm{~s}$, the data were meansmoothed using a filter kernel whose length is the length of that scale (filtering was implemented via convolution). The residual of the filtered minus the pre-filtered signal is then cut into equally spaced epochs, each with a length defined by that scale. The variance of each epoch is computed as root-mean-square, and the average variance ("fluctuations") of all epochs is computed. Finally, a least-squares fit is computed between the logarithm of these fluctuations and the logarithm of the time scales. The slope of this fit is taken as the scaling exponent. DMA 


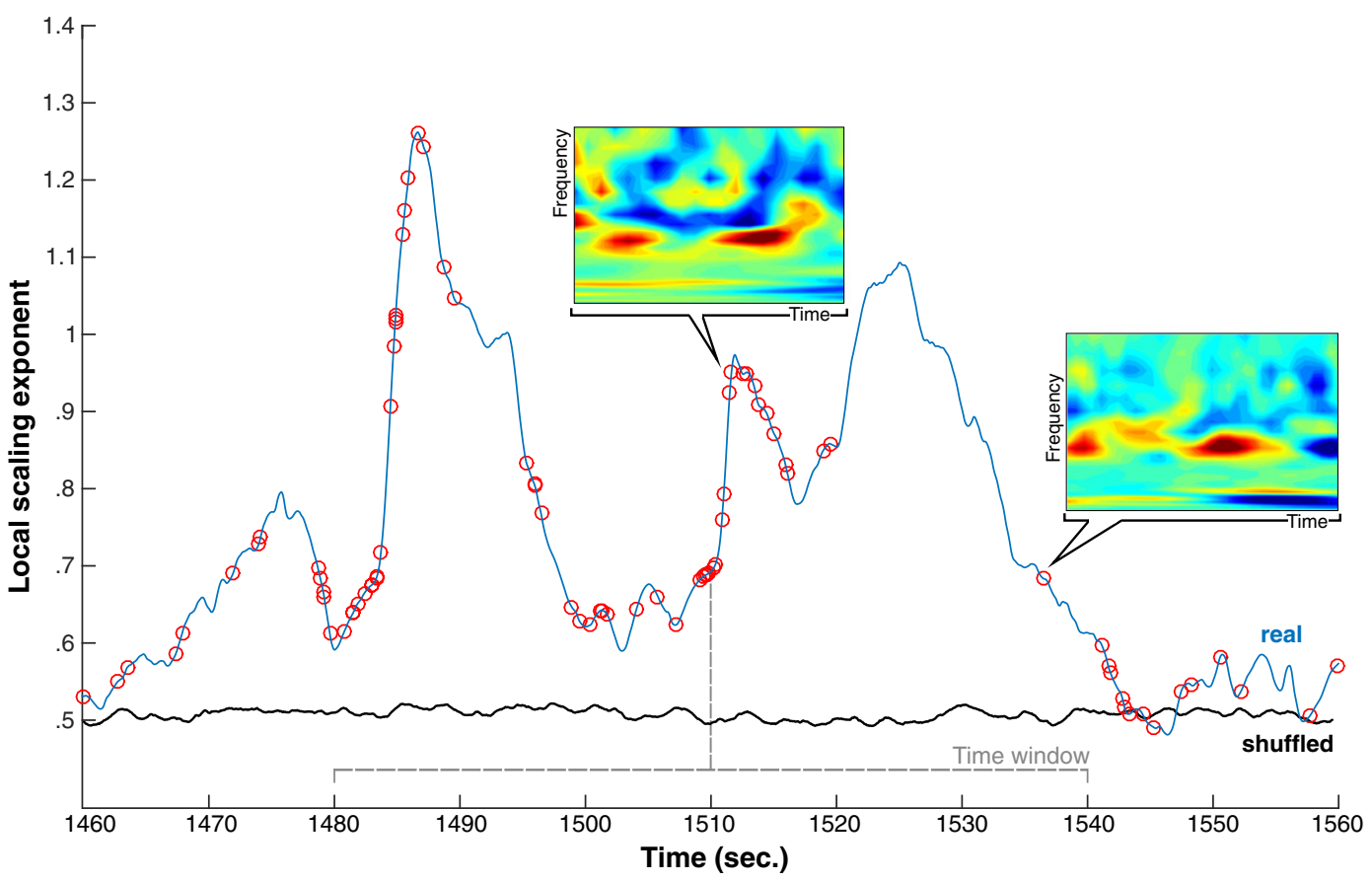

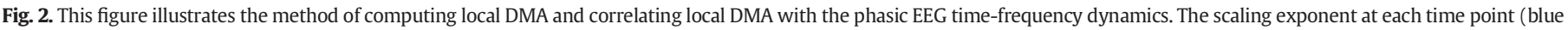

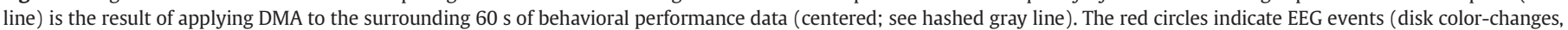

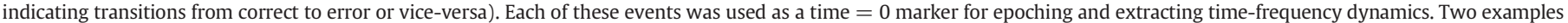

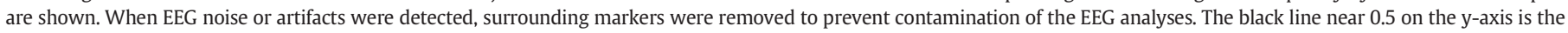

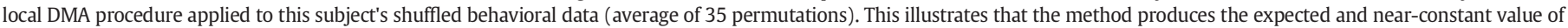
0.5 for uncorrelated noise.

of the EEG data was computed based on the power time series at each frequency band (see below for description of time-frequency decomposition method) and at each channel.

Statistical significance of the overall DMA score was evaluated against a null-hypothesis distribution of scaling exponents generated from each subject's data. For each subject, twenty random behavioral performance vectors were constructed by computing the average length of the state durations, and generating new state sequences by randomly selecting run lengths from around that average duration. Although visually these new vectors appear qualitatively similar to the empirical observations (see also Fig. S1), they do not contain longrange autocorrelated processes. Significance was then evaluated as the probability of the $Z$-normalized distance of the empirical observation related to the center of this distribution.

\section{Linking behavior and EEG activity}

In order to link the behavioral scaling exponent to phasic midfrontal theta activity within-subjects, it was necessary to obtain a "localized DMA." This was obtained by computing the scaling coefficient over shorter time windows centered on each time point (Fig. 2). There is a trade-off here, with shorter windows providing better temporal precision for the EEG analyses, but poorer estimates of scaling behavior. To determine the shortest temporal windows that would provide stable estimates of local scaling exponents, a local DMA was applied using windows ranging from 1-20 s to 1-200 s. Experiment-averaged local scaling exponents appeared to stabilize at a 1-60 s range (Fig. 1D). Furthermore, cross-subjects correlations between average local scaling exponents and the global (1-400 s) scaling exponents were statistically significant for all windows after 1-40 s (similar results between Spearman and Pearson correlations imply linear relationships that are not influenced by outliers). Correlations among results from the different local DMA windows were all strong, with many pair-wise correlations above 0.9 (Fig. S2), indicating that the local DMA estimates are robust to temporal window size. Temporal fluctuations in local DMA were not observed when applying the analysis to shuffled data (Fig. 2). Together, these results confirm that the local DMA is consistent with the global DMA, and furthermore suggest that there are temporal dynamics in local scaling behavior that are unique and missed by the global DMA result.

The analyses shown in Figs. 7 and 9 were also performed using several other time scale ranges (1-40 s, 5-60 s, 3-60 s), and the results remained qualitatively the same and statistically significant. Outliers in local DMA (defined as $>2$ standard deviations above the mean local DMA) occurred when there were too few fast-scale events to estimate fluctuations, and were removed from the analyses (results of this analysis remained statistically significant without removing outliers).

The following procedure was used to correlate the epoched EEG time-frequency data with the behavioral data (local scaling exponent, or disk diameter). First, a design matrix was created with the number of rows corresponding to the number of data epochs, and five columns corresponding to an intercept and a column for each of four conditions (line vs. flat, error vs. correct). The entries in the columns were the local behavioral DMA, or the disk diameter, at the time point of the epoch onset (time $=0$ in Figs. 5-9). The condition regressors were then $z-$ normalized. The EEG data over epochs at each time-frequency point were $z$-normalized and projected onto the design matrix as $A^{T} A \mid A^{T} B$, where $A$ is the design matrix, $B$ is the data matrix, and the back-slash is the Matlab least-squares solution to $A x=B$, and is equivalent to left-multiplying by the inverse. The solutions (beta values) were stored for each time-frequency-electrode point, for each subject. Having all conditions in the same design matrix ensures that potentially shared variance was appropriately split.

\section{EEG statistical evaluations}

Statistics were performed via permutation-based tests, which have the advantage of not making assumptions about data or parameter 
distributions, and also facilitate corrections for multiple comparisons (Cohen, 2014b; Maris and Oostenveld, 2007). At each of 10,000 iterations, the sign of the time-frequency plots (power, correlation with disk diameter, correlation with local DMA) was flipped for a random subset of subjects. This tests against the null hypothesis of zero power change or correlation coefficient. Then, a $t$-test was performed at each time-frequency pixel against zero. This provided a null-hypothesis distribution against which the observed results were compared at $\mathrm{p}<0.001$. Multiple comparisons were controlled by extracting the size of the largest supra-threshold cluster in each permutation, and accepting only those clusters in the real data that were larger than 99.9\% of this distribution (corresponding to a cluster threshold of $\mathrm{p}<0.001)$.

\section{Results}

\section{Behavioral task and results}

In the continuous action task, subjects used a mouse track-pad to follow a line on the computer monitor. In different blocks, the line was horizontal and moved up and down with fluctuating speed and random direction reversals ("flat condition"), or the line was a sinusoidal-like wave function that "waved" in addition to moving up and down ("wavey condition"). The disk was colored green while touching the line and red while off the line (respectively, correct and error) (Fig. 1A).

On average, subjects correctly tracked the line during $84.6 \%$ (standard deviation: 6.47\%) of the time for the wavey condition, and $62.5 \%$ (standard deviation: $3.27 \%$ ) of the time in the flat condition ( $\mathrm{t}_{18}=23.5, \mathrm{p}<0.001$ ). This difference in performance confirms that the task was significantly more difficult in the flat condition, consistent with the intention of allowing proactive action monitoring during the wavey condition and requiring reactive control during the flat condition.

The cumulative sum of the binary behavior time series transforms the data into an unbounded form (Fig. 1B). Qualitative inspection of these time courses suggests self-similar dynamics over at least three orders of magnitude. More formally, scale-free dynamics can be assessed via detrended or demeaned fluctuation analysis (DMA), in which the variance of the time series (root-mean-square) is computed as a function of the time window of analysis (1-400 s for consistency with previous studies). Scaling exponents $>0.5$ are taken as evidence consistent with a scale-free organization (Fig. 1C) (a scaling exponent of 0.5 indicates a random process with no "memory" or auto-correlated behavior). This average scaling exponent of all 19 subjects was 0.8233 (standard deviation 0.0245), which is in the range of scaling exponents reported in previous investigations of human task performance (Palva et al., 2013), and is statistically significantly greater than the chance level of 0.5 (all individual subject p's $<0.001$ ), assessed via simulated performance runs drawn from random distributions. These findings suggest that performance in the continuous action adjustment task followed statistical characteristics of a scale-free system with positive auto-correlated ("longterm memory") behavior.

Another method to provide evidence for this task eliciting scale-freelike behavior is to examine the distribution of time durations in each state, where "state" refers to error vs. correct (off the line vs. on the line) for the flat and wavey conditions. Histograms of these duration distributions per condition and per subject were created using 100 bins. The group average plots in log-log space exhibited a 1/f-like decrease for all conditions (Fig. S3).

\section{EEG results: overall characteristics}

EEG scaling exponents were computed over the continuous dataset (thus, over all conditions and noise-free break periods) for frequencies from 2 to $80 \mathrm{~Hz}$ and for each electrode (Hardstone et al., 2012). These findings suggest a rich landscape of long-range temporal correlations in EEG oscillatory activity, with scale-free-like patterns emerging only in some topographical regions and at some frequency ranges (Fig. 3). Higher frequencies showed generally higher scaling exponents, suggesting that broadband activity is more scale-free-like than lowerfrequency or frequency-specific activity. In fact, only in the alpha band were local peaks in scaling exponents observed, consistent with previous reports that alpha oscillatory power exhibits long-range temporal auto-correlated structure (Berthouze et al., 2010; Montez et al., 2009). No peaks were observed in the theta band, suggesting that temporal fluctuations in midfrontal theta do not exhibit long-range temporal autocorrelations consistent with scale-free-like organization (note that this does not imply that there was no theta activity; it merely shows that temporal fluctuations in theta power did not exhibit scale-freelike patterns).

\section{EEG results: cross-subjects correlations}

EEG scaling exponents were correlated with each subject's overall behavior scaling coefficient at each frequency and at each electrode. Statistics were performed by treating the data as a 2D grid and using permutation-based testing to control for multiple comparisons using cluster-correction accounting for spatial-spectral autocorrelations. When applying cluster-correction to control for multiple comparisons over electrodes, no correlations were statistically significant. The results thresholded at an uncorrected level of $\mathrm{p}<0.05$ are shown in Fig. 4. These weakly significant findings do not lend themselves to a straight-forward interpretation. They are included in the interest of completeness and comparison with other studies (e.g., Palva et al., 2013), but are not further discussed.

\section{EEG results: time-frequency characteristics}

Because midfrontal theta has not been characterized using continuous action adjustment tasks, it is important to demonstrate that the EEG results are qualitatively similar to those observed during traditional discrete-trial cognitive control tasks. Thus, EEG data were analyzed in an epoch-based manner to examine the fast-time-scale timefrequency dynamics. Four epoch types were created for each subject, corresponding to error and correct transitions for the two conditions. Results indicate a typical pattern of midfrontal theta time-locked to error onset (Fig. 5). Peak frequency was $7.56 \mathrm{~Hz}$ in both wavey error and flat error conditions. Peak times were $200 \mathrm{~ms}$ for flat errors and $250 \mathrm{~ms}$ for wavey errors. This pattern of results is important because it demonstrates that errors in this task evoke a pattern of EEG activity that is comparable with those of mistakes in other classical discretetrial tasks (e.g., flankers task, Simon task, Stroop task). Significant error-related modulations in the beta-band were also observed, though only in the flat condition.

Inter-trial phase clustering analyses revealed little "phase reset" by the condition onsets, and virtually no phase clustering in the theta band (Fig. 6). In other words, the theta dynamics reflected amplitudemodulations of ongoing rhythmic activity, as opposed to additive or phase-aligned evoked responses. This finding is also consistent with previous methodological investigations that have revealed little functional importance of the phase-locked component of midfrontal theta (Cohen and Donner, 2013), although other studies have highlighted that the phase-locked component of error-related activity may also provide some unique information (Munneke et al., 2015; Trujillo and Allen, 2007).

\section{EEG results: within-subjects}

The results so far demonstrate that behavioral performance during online action adjustments is organized over a range of time scales 

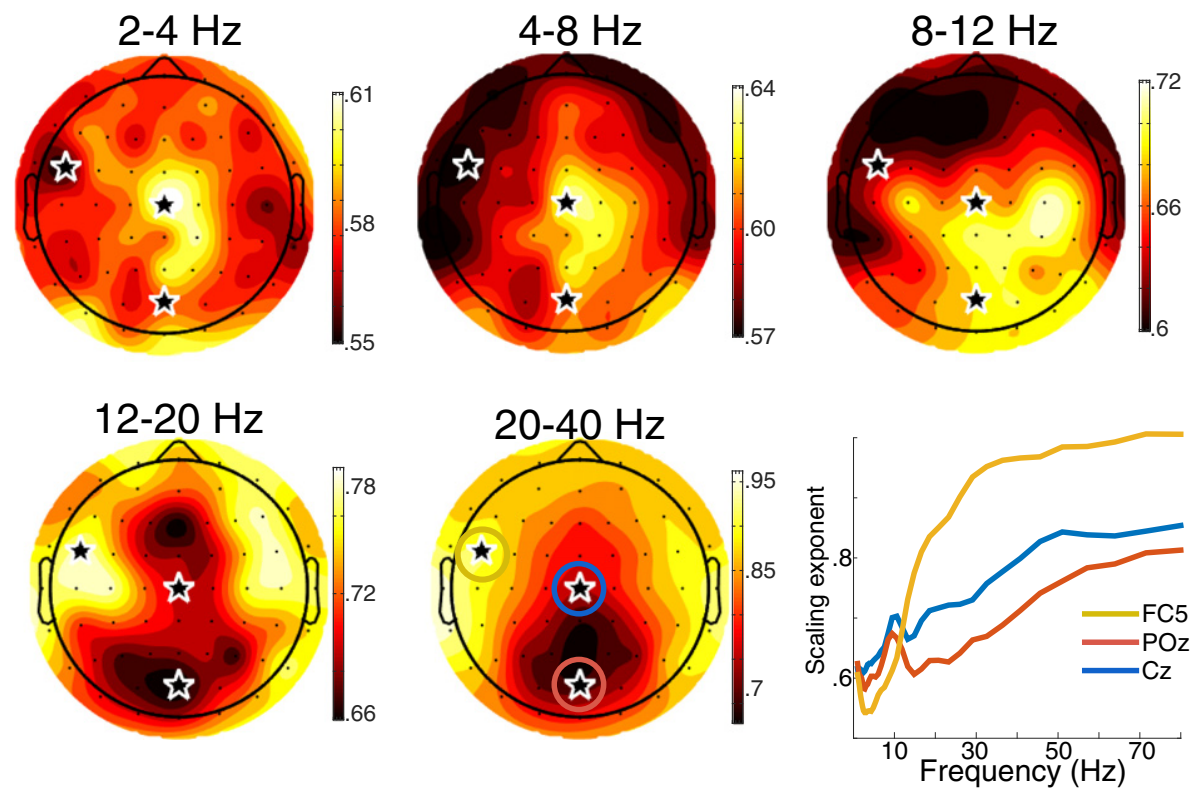

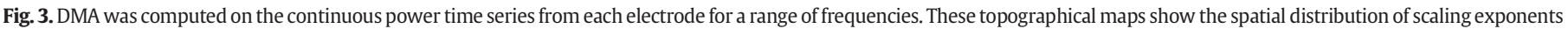
from different frequency ranges, with results from three electrodes highlighted in the lower-left panel.

from seconds to hundreds of seconds, and that neural signatures of task performance are observed at a fast time-scale of a few hundred ms. Are these multiscale dynamics related to each other? To address this question, the temporally localized adaptation of the DMA was correlated with the phasic EEG results using within-subjects regression analyses. Note that in these analyses, the local scaling exponent time series was generated from the behavioral data, not from the EEG data. This eliminates any possible biases of temporal smoothing or of circular analyses that could arise if the local DMA was correlated with the same data from which it was estimated.
The temporally localized behavioral scaling exponent was extracted at each time $=0$ point in the plots in Fig. 5 (in other words, each time the subject initiated an error or a correction). Local scaling exponents were then correlated with EEG time-frequency power over trials (Fig. 2 ). Results are presented in Fig. 7. In these plots, the color at each time-frequency pixel corresponds to the subject-average correlation coefficient between power over epochs and the behavioral local scaling exponent at the time of the color change. In the flat error condition, there appears to be two significant regions around the theta band, one in the classical theta band (4.8 to $9.4 \mathrm{~Hz}$ ) and another in the upper
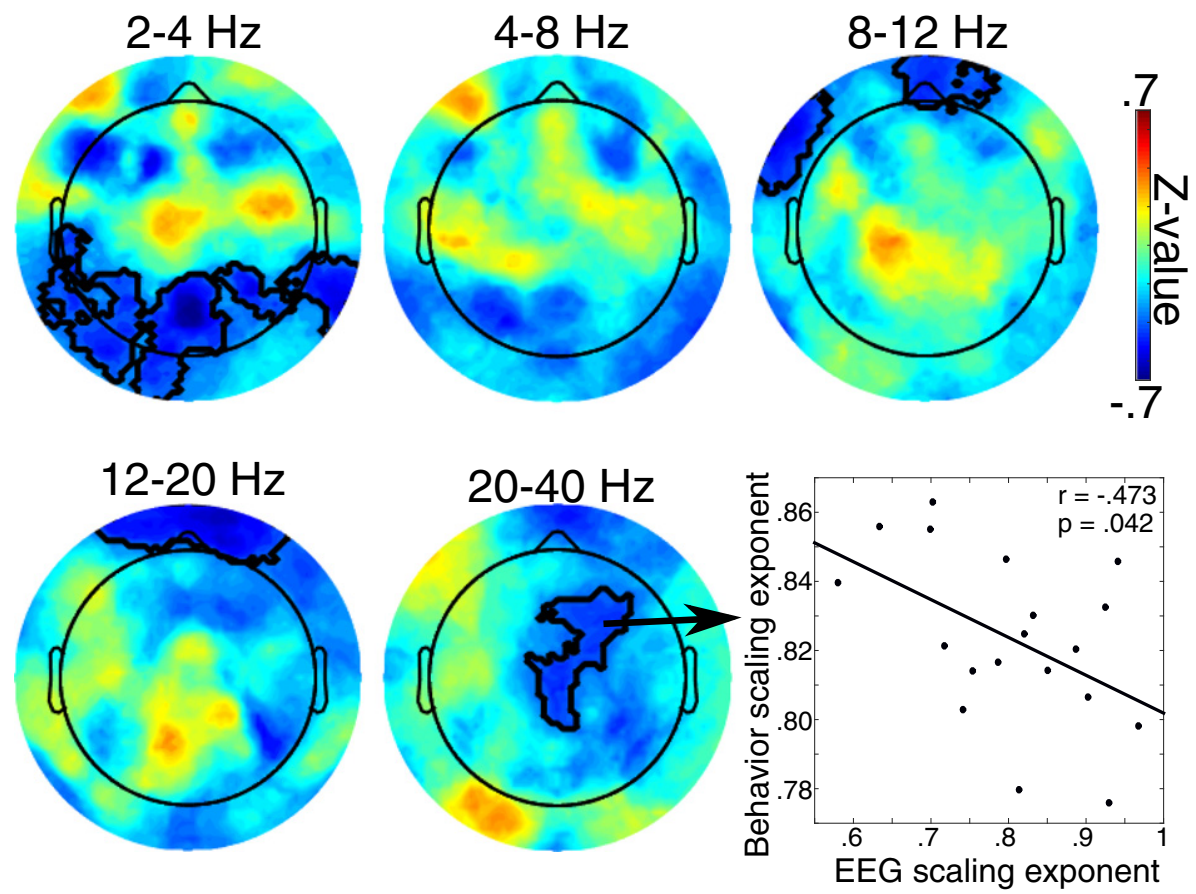

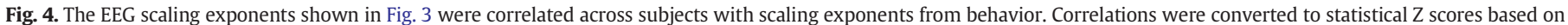

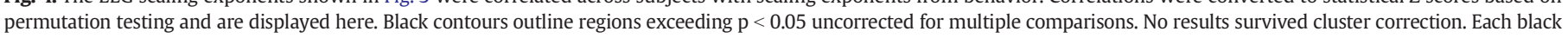
dot in the lower-right panel is a subject. 

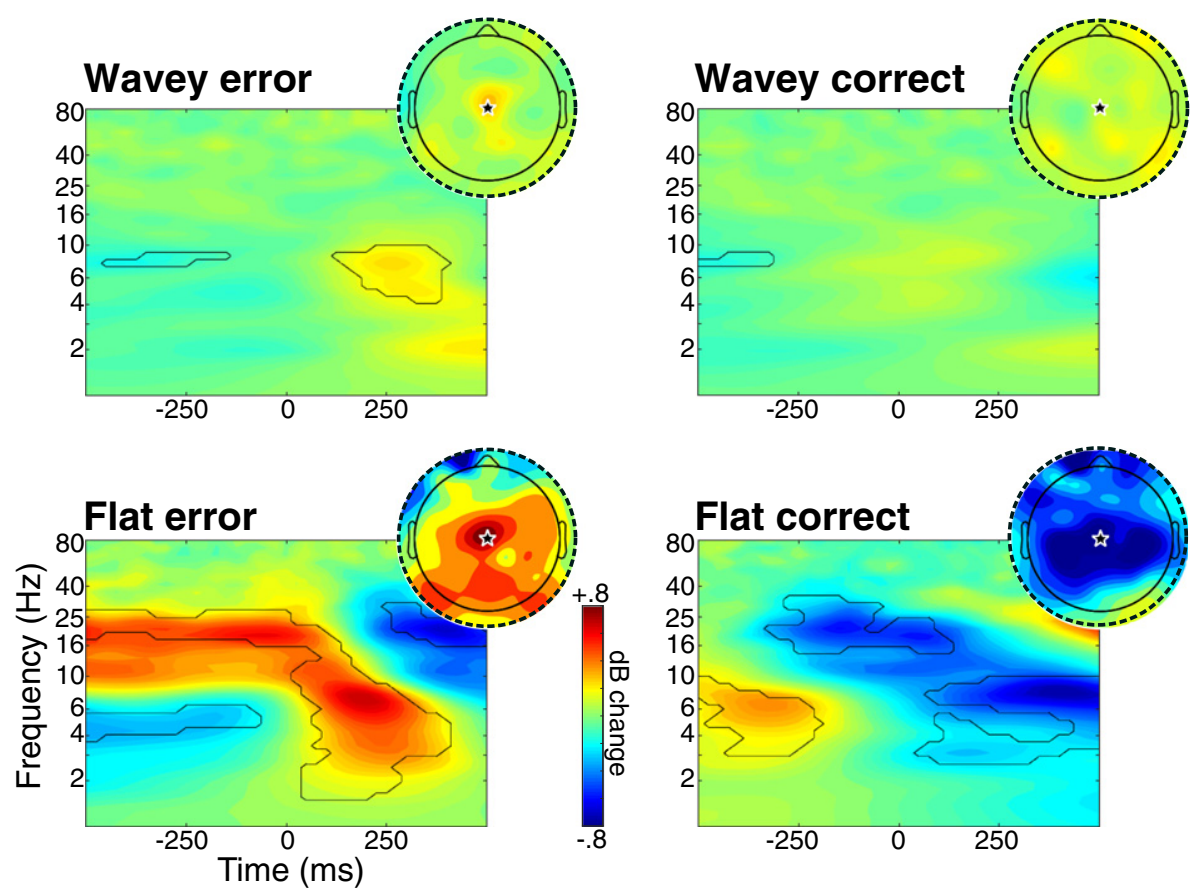

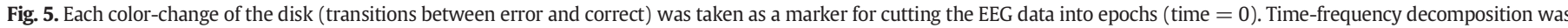

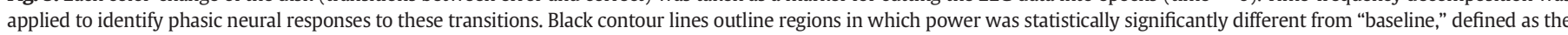

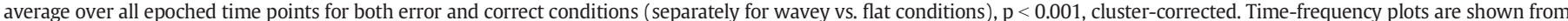

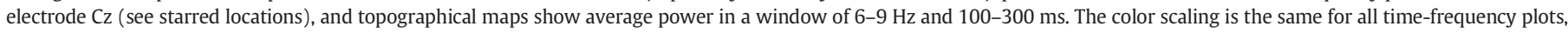
and scaled by 0.5 for the topographical maps.

delta band (2.2 to $3.8 \mathrm{~Hz}$ ). This distinction may reflect statistical thresholding rather than truly distinct frequency components, so the two blobs are interpreted as reflecting a single theta-band component (indeed, there is only a single supra-threshold theta peak preceding correct responses). The peak time-frequency point was $6.76 \mathrm{~Hz}$ at $200 \mathrm{~ms}$. The time-frequency-topographical characteristics of this correlation were similar to the overall theta power effect (i.e., compare the theta- band activity in Fig. 7 with Fig. 5), suggesting that these correlations may reflect behavioral modulations of the phasic theta dynamics shown in Fig. 5.

Perhaps this correlation is due to the limited time range used for the local DMA, such that the result simply reflects average task performance over the previous few seconds. Fortunately, the disk diameter provided a useful control analysis: disk diameter reflected the unweighted
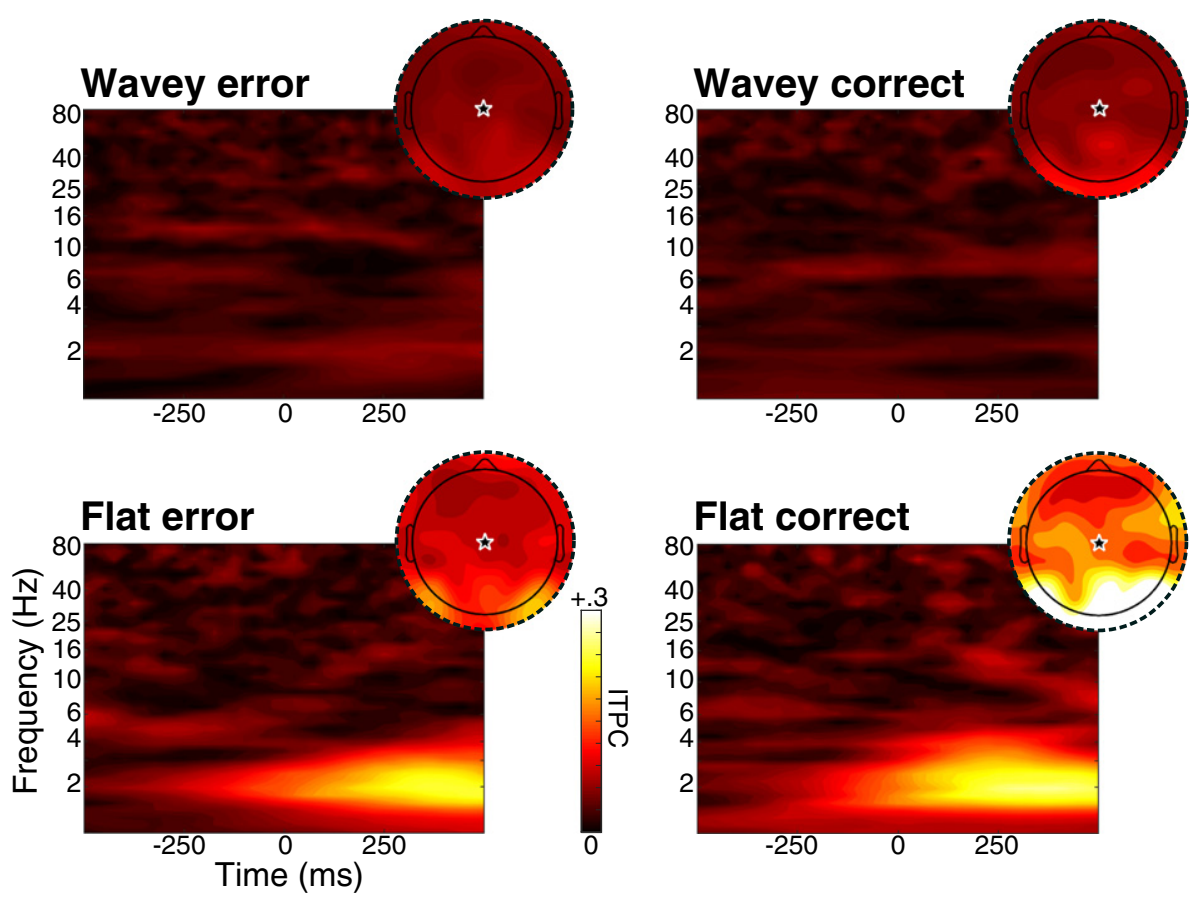

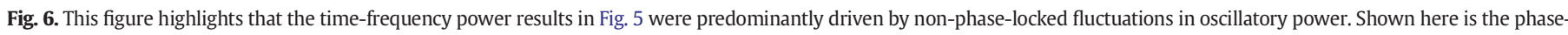
consistency over trials for each condition. 

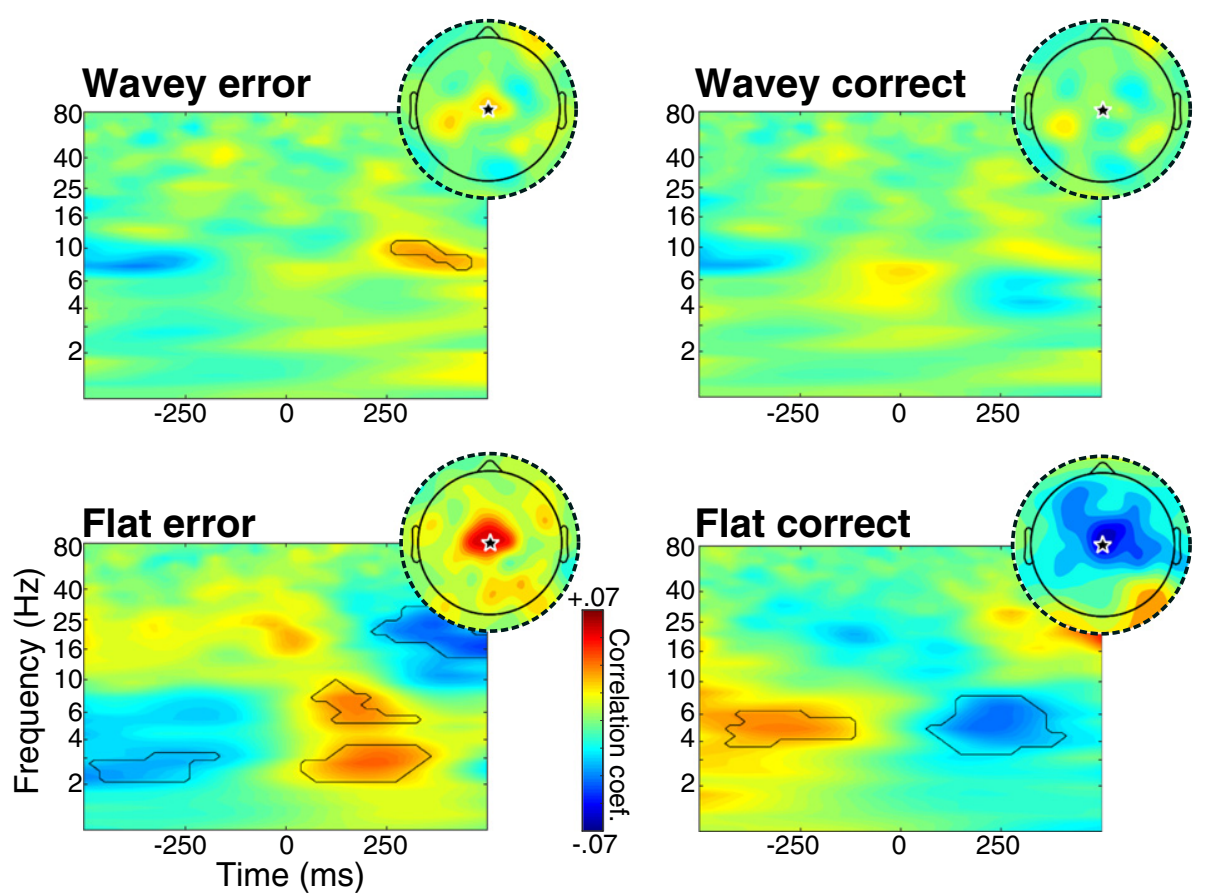

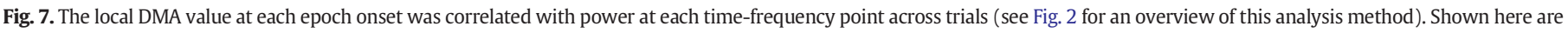

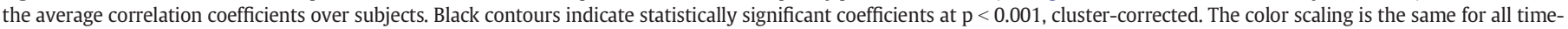
frequency plots, and scaled by 0.5 for the topographical maps.

behavioral performance over a fixed time-scale of the 5 preceding seconds (disk diameter was titrated according to the proportion of time spent off the line). Therefore, disk diameter was used in the same within-subjects correlation analysis that was used to link the local scaling to EEG.

Interestingly, disk diameter was also significantly correlated with theta-band power (Fig. 8), but with different temporal characteristics compared to the correlations with local scaling exponents. The correlations were observed in all conditions, and peaked earlier in time. A comparison between the EEG-disk diameter and the EEG-local scaling exponent correlations can be seen in Fig. 9. In this figure, the timecourses of the correlation coefficients for each subject in the theta band are shown (averaged from 3 to $8 \mathrm{~Hz}$ ). The subject-average correlation coefficient time course plots reveal the differential timing between how action adjustment-related midfrontal theta was related to local behavioral scaling vs. the disk-diameter. They also show that the withinsubjects correlations, though small in magnitude, are highly consistent over subjects.
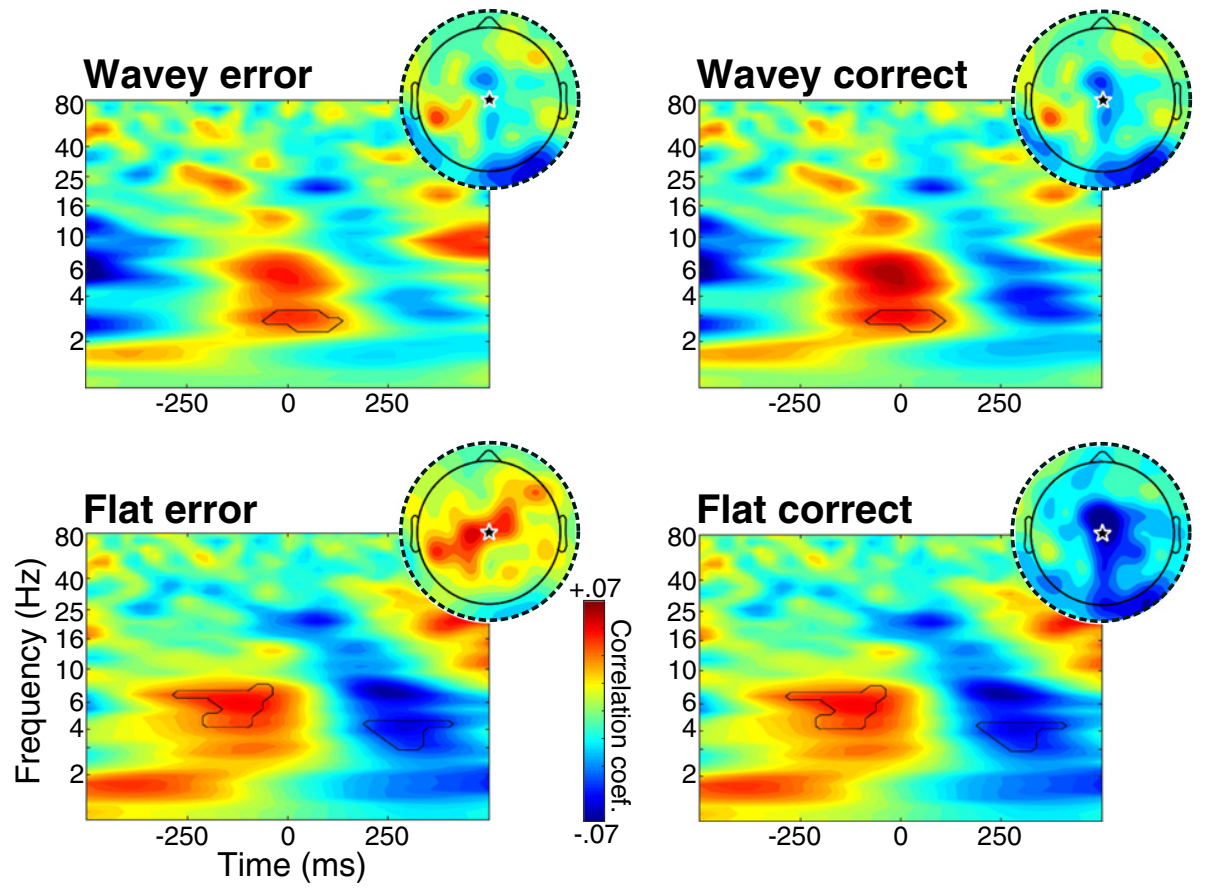

Fig. 8. The same analysis as in Fig. 7 was applied using the disk diameter as the independent variable instead of local DMA. 
Local DMA-theta correlations

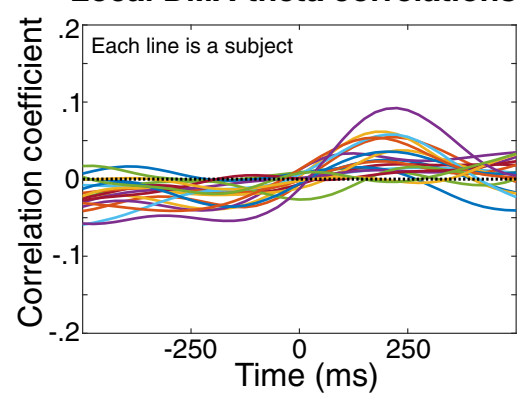

Disk diameter-theta correlations

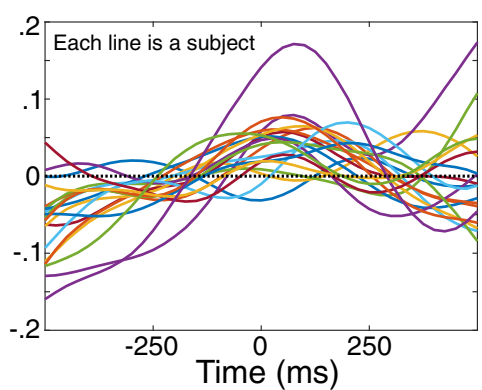

Comparison of time courses

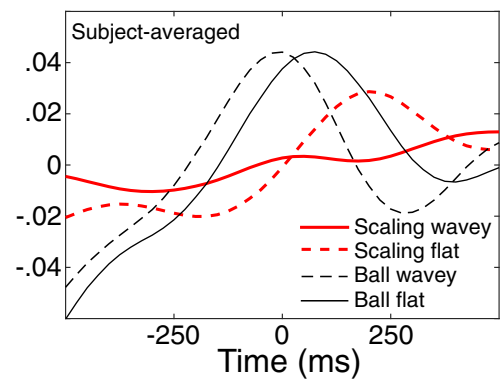

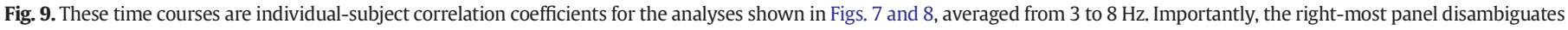
the correlation time courses, illustrating that the theta-local DMA correlations are distinct from the theta-disk diameter correlations.

Taken together, these findings suggest that the EEG-scalingexponent correlations are attributable to fluctuations in scaling exponents in behavior over tens of seconds, and are not confounded by fixed-time-scale performance indicators over $5 \mathrm{~s}$. Indeed, it seems that the midfrontal theta response-which so far has been studied only in the phasic time-scale of hundreds of milliseconds-is sensitive to task performance over multiple longer time scales.

\section{Discussion}

The relationship between human EEG activity and action monitoring has been studied exclusively using one time scale: a few hundreds of milliseconds between stimulus onset and manual response. Over the past 25 years, this approach has led to many important findings, including the initial identification of a phasic event-related potential to response conflict and errors, the observation of non-phase-locked oscillations, large-scale cortico-cortical and cortico-subcortical synchronization, and links to psychopathologies and clinical conditions including obsessive-compulsive disorder and Parkinson's disease (Cavanagh and Frank, 2014; Cavanagh and Shackman, n.d.; Cohen et al., 2008; Gehring et al., 2012). The contribution of the present study is to demonstrate that this phasic midfrontal theta-band response to action monitoring and error correction is modulated by behavioral dynamics over a broad range of time scales spanning at least three orders of magnitude, from hundreds of milliseconds to a few seconds to scale-free-like patterns over several tens of seconds.

What are these slower and long-range temporally autocorrelated processes, and what causes them? The answers to both questions are uncertain. They may reflect a combination of fluctuations in task engagement or sustained attention, and fluctuations in neurochemical and hormonal release. Indeed, slow fluctuations in brain activity are known to predict mistakes in attention tasks, in some cases several tens of seconds before the error (Eichele et al., 2008; O'Connell et al., 2009).

Scale-free-like dynamics in brain structure and function are taken as evidence for the brain operating in a criticality regime (Beggs and Plenz, 2003; Linkenkaer-Hansen et al., 2001). Operating at a critical or nearcritical state may help maximize the flexibility to adapt to sudden changes in the environment (Chialvo, 2010). On the other hand, the literature on midfrontal theta consistently demonstrates that midfrontal theta is a signature of the rapid engagement of an adaptive system that flexibly responds to errors or the possibility of errors (Cavanagh and Frank, 2014; Cohen, 2014a; Gehring et al., 2012). The present study is the first to link these two literatures by demonstrating that the phasic brain response to errors and error corrections is modulated by slow fluctuations in criticality as measured by task performance.

Computational modeling studies demonstrate that certain types of neural network configurations can produce scale-free-like or selforganized activity (Poil et al., 2012; Priesemann et al., 2014). On the other hand, midfrontal theta power did not exhibit robust signatures of scale-free-like activity. It is possible that midfrontal theta would exhibit scale-free-like patterns during longer periods of rest (e.g., Euler et al., 2016), and that the task engagement disrupts these long-range temporal autocorrelations. Nonetheless, the absence of scale-free-like organization of midfrontal theta makes the phasic theta modulation by behavioral local scaling particularly interesting. It is tempting to speculate that slower fluctuations in scale-free-like patterns provide a background milieu within which phasic neural responses are computed. Precisely what causes these fluctuations in background milieu and how it affects the neural computations that manifest as midfrontal theta is an open question. But clearly it is not a simple matter of generic brain responses being yoked to behavioral temporal dynamics, because these correlations were specific in time, frequency, space, and condition. Instead, it seems that these modulations are present primarily when reactive control is needed.

Another important finding of this study is that the EEG responses to errors and their corrections were broadly consistent with findings obtained in typical cognitive control tasks. The effect sizes (in terms of $\mathrm{dB}$-normalized power) were generally smaller than in discrete-trial tasks, but this should not be very surprising: Typical tasks have many components at each trial onset, including orienting, attention, and working memory (to recall the task instructions and learned rules). This and other recent studies (Cavanagh and Castellanos, 2016) demonstrate that the classic computerized tasks used in this field can be supplemented by more real-world relevant tasks.

Furthermore, in most tasks that are used to study executive functioning, errors are rare and thus elicit additional processes related to novelty detection, arousal, and emotion (Notebaert et al., 2009; Wessel et al., 2012). The present task avoids these additional factors (for example, there were an equal number of error onsets and corrections), and thus allows the findings to reflect more uncontaminated action monitoring and adjustment properties. The topography of the error-correct effect, however, was slightly more posterior than seen in other studies, which tend to show maximal error-correct activity around FCz. It is not immediately clear why the topography shifted posterior, but this may be due to the strong motoric aspects of the present task.

The theta dynamics were more apparent during the flat condition compared to the wavey condition (Fig. 5). This matches the goal of the manipulation, which was to force subjects to utilize a reactive control strategy (flat condition, with unpredictable movements) or a proactive control strategy (wavey condition, with predictable movements). Previous studies have shown that frontal EEG responses are stronger, and sometimes only observed, during trials that require reactive control (Bartholow et al., 2005; Folstein and Van Petten, 2008). Furthermore, action monitoring-related midfrontal theta appears to be particularly sensitive to reactive control that is driven by visual-spatial properties, such as in the "Simon task" (Leuthold, 2011), and in the present task.

What have we learned about midfrontal theta and response conflict from this study? One of the important questions in this literature is 
whether midfrontal theta plays a meaningful role in the neural computations underlying action monitoring, and if so, what is the computational significance of theta? Recent evidence using rhythmic electrical brain stimulation supports the idea that theta plays a causal role in these processes (van Driel et al., 2015). Unfortunately, the present results provide no new insights into the possible causal role of the theta rhythm in the neural computations underlying action monitoring. However, these results demonstrate that midfrontal theta is sensitive to subtle fluctuations in behavior over multiple time-scales, which provides a tighter link between the EEG signal and online action adjustments, beyond simple correlations with reaction times. Perhaps the most important aspects of this study are forming a bridge between previously disparate literatures on scale-free dynamics and action monitoring, and demonstrating that phasic neural responses to cognitive-motor demands are more sensitive than previously thought to larger and longer ongoing behavioral state fluctuations. Slow fluctuations in metastability and criticality seem to modulate the phasic computations that underlie rapid online error corrections. Discovering why this is the case may shed new light onto the functional significance and possible clinical relevance of cognitive control-related midfrontal theta.

\section{Competing interests}

None.

\section{Funding}

MXC is funded by an ERC-StG 638589.

\section{Acknowledgments}

Thanks to Katerina Georgopoulou for collecting the data during her master's thesis project. Thanks to Eric Maris for comments on the manuscript.

\section{Appendix A. Supplementary data}

Supplementary data to this article can be found online at http://dx. doi.org/10.1016/j.neuroimage.2016.07.054.

\section{References}

Akam, T.E., Kullmann, D.M. 2012. Efficient “communication through coherence” requires oscillations structured to minimize interference between signals. PLoS Comput. Biol. 8 (11), e1002760 (http://doi.org/10.1371/journal.pcbi.1002760)

Bartholow, B.D., Pearson, M.A., Dickter, C.L., Sher, K.J., Fabiani, M., Gratton, G., 2005. Strategic control and medial frontal negativity: beyond errors and response conflict. Psychophysiology 42 (1), 33-42 (http://doi.org/10.1111/j.1469-8986.2005.00258.x).

Beggs, J.M., Plenz, D., 2003. Neuronal avalanches in neocortical circuits. The Journal of Neuroscience 23 (35), 11167-11177 (Retrieved from http://www.ncbi.nlm.nih.gov/ pubmed/14657176)

Beggs, J.M., Timme, N., 2012. Being critical of criticality in the brain. Front. Physiol. 3, 163 (http://doi.org/10.3389/fphys.2012.00163).

Berthouze, L., James, L.M., Farmer, S.F., 2010. Human EEG shows long-range temporal correlations of oscillation amplitude in Theta, Alpha and Beta bands across a wide age range. Clinical Neurophysiology 121 (8), 1187-1197 (http://doi.org/10.1016/j. clinph.2010.02.163).

Buzsáki, G., Moser, E.I., 2013. Memory, navigation and theta rhythm in the hippocampalentorhinal system. Nature Neuroscience 16 (2), 130-138 (http://doi.org/10.1038/nn 3304).

Cavanagh, J.F., Castellanos, J., 2016. Identification of canonical neural events during continuous gameplay of an 8-bit style video game. NeuroImage 133, 1-13.

Cavanagh, J.F., Frank, M.J., 2014. Frontal theta as a mechanism for cognitive control. Trends Cogn. Sci. 18 (8), 414-421 (http://doi.org/10.1016/j.tics.2014.04.012).

Cavanagh, J. F., \& Shackman, A. J. Frontal midline theta reflects anxiety and cognitive control: meta-analytic evidence. Journal of Physiology, Paris, 109(1-3), 3-15. (http://doi. org/10.1016/j.jphysparis.2014.04.003)

Chialvo, D.R., 2010. Emergent complex neural dynamics. Nat. Phys. 6 (10), 744-750 (http://doi.org/10.1038/nphys1803).

Cohen, M.X., 2011. Error-related medial frontal theta activity predicts cingulate-related structural connectivity. NeuroImage 55 (3), 1373-1383 (Retrieved from http:// www.ncbi.nlm.nih.gov/pubmed/21195774).
Cohen, M.X., 2014a. A neural microcircuit for cognitive conflict. Trends Neurosci. 37 (9), 480-490.

Cohen, M.X., 2014b. Analyzing Neural Time Series Data: Theory and Practice. MIT Press, Cambridge.

Cohen, M.X., Donner, T.H., 2013. Midfrontal conflict-related theta-band power reflects neural oscillations that predict behavior. J. Neurophysiol. 110 (12), 2752-2763 (http://doi.org/10.1152/jn.00479.2013).

Cohen, MX., Ridderinkhof, K.R., Haupt, S., Elger, C.E., Fell, J., 2008. Medial frontal cortex and response conflict: evidence from human intracranial EEG and medial frontal cortex lesion. Brain Res. 1238, 127-142 (http://doi.org/10.1016/j.brainres.2008.07.114).

Delorme, A., Makeig, S., 2004. EEGLAB: an open source toolbox for analysis of single-trial EEG dynamics including independent component analysis. J. Neurosci. Methods 134 (1), 9-21 (http://doi.org/10.1016/j.jneumeth.2003.10.009).

Donner, T.H., Siegel, M., 2011. A framework for local cortical oscillation patterns. Trends Cogn. Sci. 15 (5), 191-199 (http://doi.org/10.1016/j.tics.2011.03.007).

Egner, T., 2007. Congruency sequence effects and cognitive control. Cognitive, Affective \& Behavioral Neuroscience 7 (4), 380-390 (Retrieved from http://www.ncbi.nlm.nih. gov/pubmed/18189011)

Egner, T., Ely, S., Grinband, J., 2010. Going, going, gone: characterizing the time-course of congruency sequence effects. Front. Psychol. 1, 154 (http://doi.org/10.3389/fpsyg. 2010.00154).

Eichele, T., Debener, S., Calhoun, V.D., Specht, K., Engel, A.K., Hugdahl, K., ... Ullsperger, M., 2008. Prediction of human errors by maladaptive changes in event-related brain networks. Proc. Natl. Acad. Sci. U. S. A. 105 (16), 6173-6178 (http://doi.org/10.1073/ pnas.0708965105).

Euler, M.J., Wiltshire, T.J., Niermeyer, M.A., Butner, J.E., 2016. Working memory performance inversely predicts spontaneous delta and theta-band scaling relations. Brain Research 1637, 22-33. http://dx.doi.org/10.1016/j.brainres.2016.02.008.

Farrell, S., Wagenmakers, E.-J., Ratcliff, R., 2006. 1/f noise in human cognition: is it ubiquitous, and what does it mean? Psychonomic Bulletin \& Review 13 (4), 737-741 (Retrieved from http://www.ncbi.nlm.nih.gov/pubmed/17201378).

Folstein, J.R., Van Petten, C., 2008. Influence of cognitive control and mismatch on the N2 component of the ERP: a review. Psychophysiology 45 (1), 152-170 (http://doi.org/ 10.1111/j.1469-8986.2007.00602.x).

Fries, P. (2005). A mechanism for cognitive dynamics: Neuronal communication through neuronal coherence. Trends in Cognitive Sciences. (http://doi.org/10.1016/ j.tics.2005.08.011)

Gehring, W.J., Liu, Y., Orr, J.M., Carp, J., 2012. The Error-Related Negativity (ERN/Ne). In: Luck, S.J., Kappenman, E.S. (Eds.), The Oxford Handbook of Event-Related Potentials. Oxford University Press, New York, pp. 231-291

Hardstone, R., Poil, S.-S., Schiavone, G., Jansen, R., Nikulin, V.V., Mansvelder, H.D., Linkenkaer-Hansen, K., 2012. Detrended fluctuation analysis: a scale-free view on neuronal oscillations. Front. Physiol. 3, 450 (http://doi.org/10.3389/fphys.2012.00450).

Huster, R.J., Enriquez-Geppert, S., Lavallee, C.F., Falkenstein, M., Herrmann, C.S., 2013. Electroencephalography of response inhibition tasks: Functional networks and cognitive contributions. International Journal of Psychophysiology 87 (3), 217-233. http:// dx.doi.org/10.1016/j.ijpsycho.2012.08.001.

Ihlen, E.A.F., 2012. Introduction to multifractal detrended fluctuation analysis in matlab. Front. Physiol. 3, 141 (http://doi.org/10.3389/fphys.2012.00141).

Jiang, Z.-Q., Zhou, W.-X., 2011. Multifractal detrending moving-average cross-correlation analysis. Physical Review. E, Statistical, Nonlinear, and Soft Matter Physics 84 (1 Pt 2), 016106 (http://doi.org/10.1103/PhysRevE.84.016106).

Kello, C.T., Brown, G.D.A., Ferrer-I-Cancho, R., Holden, J.G., Linkenkaer-Hansen, K., Rhodes, T., Van Orden, G.C., 2010. Scaling laws in cognitive sciences. Trends Cogn. Sci. 14 (5), 223-232 (http://doi.org/10.1016/j.tics.2010.02.005)

Le Van Quyen, M., Botella-Soler, V., Valderrama, M., 2013. Neuronal oscillations scale up and scale down brain dynamics. In: Pesenson, M.M.Z. (Ed.), Multiscale Analysis and Nonlinear Dynamics. Wiley-VCH Verlag GmbH \& Co. KGaA, Weinheim, Germany, pp. 147-177 (http://doi.org/10.1002/9783527671632).

Leuthold, H., 2011. The Simon effect in cognitive electrophysiology: a short review. Acta Psychol. 136 (2), 203-211 (http://doi.org/10.1016/j.actpsy.2010.08.001)

Linkenkaer-Hansen, K., Nikouline, V.V., Palva, J.M., Ilmoniemi, R.J., 2001. Long-range temporal correlations and scaling behavior in human brain oscillations. The Journal of Neuroscience 21 (4), 1370-1377 (Retrieved from http://www.ncbi.nlm.nih.gov/ pubmed/11160408).

Maris, E., Oostenveld, R., 2007. Nonparametric statistical testing of EEG- and MEG-data. J. Neurosci. Methods 164 (1), 177-190 (http://doi.org/10.1016/j.jneumeth.2007.03. 024).

Montez, T., Poil, S.-S., Jones, B.F., Manshanden, I., Verbunt, J.P.A., van Dijk, B.W., ... Linkenkaer-Hansen, K., 2009. Altered temporal correlations in parietal alpha and prefrontal theta oscillations in early-stage Alzheimer disease. Proc. Natl. Acad. Sci. U. S. A. 106 (5), 1614-1619 (http://doi.org/10.1073/pnas.0811699106).

Munneke, G.-J., Nap, T.S., Schippers, E.E., Cohen, M.X., 2015. A statistical comparison of EEG time- and time-frequency domain representations of error processing. Brain Res. 1618, 222-230 (http://doi.org/10.1016/j.brainres.2015.05.030).

Nakao, T., Bai, Y., Nashiwa, H., Northoff, G., 2012. Resting-state EEG power predicts conflictrelated brain activity in internally guided but not in externally guided decisionmaking. Neurolmage 66C, 9-21 (http://doi.org/10.1016/j.neuroimage.2012.10.034).

Narayanan, N.S., Cavanagh, J.F., Frank, M.J., Laubach, M., 2013. Common medial frontal mechanisms of adaptive control in humans and rodents. Nat. Neurosci. 16 (12), 1888-1895 (http://doi.org/10.1038/nn.3549).

Nigbur, R., Cohen, M.X., Ridderinkhof, K.R., Stürmer, B., 2012. Theta dynamics reveal domain-specific control over stimulus and response conflict. Journal of Cognitive Neuroscience 24 (5), 1264-1274 (http://doi.org/10.1162/jocn_a_00128). 
Notebaert, W., Houtman, F., Opstal, F.V., Gevers, W., Fias, W., Verguts, T., 2009. Post-error slowing: an orienting account. Cognition 111 (2), 275-279 (http://doi.org/10.1016/j. cognition.2009.02.002)

O'Connell, R.G., Dockree, P.M., Robertson, I.H., Bellgrove, M.A., Foxe, J.J., Kelly, S.P., 2009. Uncovering the neural signature of lapsing attention: electrophysiological signals predict errors up to $20 \mathrm{~s}$ before they occur. J. Neurosci. Off. J. Soc. Neurosci. 29 (26), 8604-8611 (http://doi.org/10.1523/JNEUROSCI.5967-08.2009).

Oehrn, C.R., Hanslmayr, S., Fell, J., Deuker, L., Kremers, N.A., Do Lam, A.T., ... Axmacher, N., 2014. Neural communication patterns underlying conflict detection, resolution, and adaptation. The Journal of Neuroscience 34 (31), 10438-10452 (http://doi.org/10. 1523/JNEUROSCI.3099-13.2014).

Palva, J.M., Zhigalov, A., Hirvonen, J., Korhonen, O., Linkenkaer-Hansen, K., Palva, S., 2013. Neuronal long-range temporal correlations and avalanche dynamics are correlated with behavioral scaling laws. Proc. Natl. Acad. Sci. U. S. A. 110 (9), 3585-3590 (http://doi.org/10.1073/pnas.1216855110).

Poil, S.-S., Hardstone, R., Mansvelder, H.D., Linkenkaer-Hansen, K., 2012. Critical-state dynamics of avalanches and oscillations jointly emerge from balanced excitation/inhibition in neuronal networks. The Journal of Neuroscience 32 (29), 9817-9823 (http:// doi.org/10.1523/JNEUROSCI.5990-11.2012)

Priesemann, V., Wibral, M., Valderrama, M., Pröpper, R., Le Van Quyen, M., Geisel, T., ... Munk, M.H.J., 2014. Spike avalanches in vivo suggest a driven, slightly subcritical brain state. Front. Syst. Neurosci. 8, 108 (http://doi.org/10.3389/fnsys.2014.00108).

Shao, Y.-H., Gu, G.-F., Jiang, Z.-Q., Zhou, W.-X., Sornette, D., 2012. Comparing the performance of FA, DFA and DMA using different synthetic long-range correlated time series. Scientific Reports 2, 835 (http://doi.org/10.1038/srep00835).
Stokić, M., Milovanović, D., Ljubisavljević, M.R., Nenadović, V., Čukić, M., 2015. Memory load effect in auditory-verbal short-term memory task: EEG fractal and spectral analysis. Exp. Brain Res. 233 (10), 3023-3038 (http://doi.org/10.1007/s00221-015-4372-z).

Trujillo, L.T., Allen, J.J.B., 2007. Theta EEG dynamics of the error-related negativity. Clinica Neurophysiology 118 (3), 645-668 (http://doi.org/10.1016/j.clinph.2006.11.009).

van Driel, J., Sligte, I.G., Linders, J., Elport, D., Cohen, M.X., 2015. Frequency band-specific electrical brain stimulation modulates cognitive control processes. PLoS One 10 (9) e0138984 (http://doi.org/10.1371/journal.pone.0138984)

Varela, F., Lachaux, J.P., Rodriguez, E., Martinerie, J., 2001. The brainweb: phase synchronization and large-scale integration. Nature Reviews. Neuroscience 2 (4), 229-239 (http://doi.org/10.1038/35067550).

Wang, X.-J., 2010. Neurophysiological and computational principles of cortical rhythms in cognition. Physiol. Rev. 90 (3), 1195-1268 (http://doi.org/10.1152/physrev.00035. 2008).

Wessel, J.R., Danielmeier, C., Morton, J.B., Ullsperger, M., 2012. Surprise and error: common neuronal architecture for the processing of errors and novelty. J. Neurosci. Off. J. Soc. Neurosci. 32 (22), 7528-7537 (http://doi.org/10.1523/JNEUROSCI.6352-11.2012).

Womelsdorf, T., Johnston, K., Vinck, M., Everling, S., 2010. Theta-activity in anterior cingulate cortex predicts task rules and their adjustments following errors. Proc. Natl. Acad. Sci. 107 (11), 5248-5253 (http://doi.org/10.1073/pnas.0906194107). 